\title{
Agent Based Disaster Evacuation Assistance System
}

\author{
Yasuki Iizuka *, Katsuya Kinoshita ${ }^{\dagger}$, Kayo Iizuka ${ }^{\ddagger}$
}

\begin{abstract}
This paper proposes a system that supports people being evacuated effectively from dangerous situations by using multi-agent cooperation. The main feature of this system is that it does not require central servers. The system uses the mobile devices of evacuees (e.g. phones, mobile PCs, tablets) and performs distributed calculations while assessing the locations of evacuees. By using this system, the evacuees are able to know the appropriate evacuation timing. This paper focuses on the formalization of the disaster evacuation problem and how to solve it using the framework of the Distributed Constraint Optimization Problem (DCOP). In order to evaluate this system, an experiment was carried out using multi-agent simulation. The result of the experiment showed that, for the case where the evacuees can receive evacuation guidance from this system, the evacuation completion time for all evacuees (in the case) was about 10\% - 30\% less than in the case where this system is not used.
\end{abstract}

Keywords: disaster evacuation, multi-agent simulation, DCOP

\section{Introduction}

In times of disaster, or other emergent situations, it is critical for people to be evacuated in a smooth manner. However, this is not easy to realize, because people often tend to panic when faced with disaster, crowding the evacuation passageways of buildings in the event of fire and congesting roads with cars containing people fleeing from predicted hurricanes. On the other hand, people do not attempt to evacuate themselves from danger when the normalcy bias has occurred, and this also makes it difficult to realize an effective and smooth evacuation. Therefore, evacuation guidance is very important. However, the disaster countermeasures office would be unable to guide all evacuees thoroughly at the time of any disaster. Evacuees need to take refuge based on mutual-help [1].

To deal with this issue, we propose a system that provides optimal evacuation guidance autonomously at the time of a disaster. The system works on the mobile devices of evacuees, performs distributed calculations using the framework of the Distributed Constraint Optimization Problem (DCOP) [2], and does not need a central server.

* Tokai University, Kanagawa, Japan

† Tokai University, Kanagawa, Japan, System Research Company Limited, Aichi, Japan

* Senshu University, Kanagawa, Japan 
This paper presents an overview and the evaluation results of the prototype of the disaster evacuation assistance system. The functions required for evacuation systems are described in Section 2. This paper focuses on the formalization of the disaster evacuation problem, and how to solve it using the framework of the DCOP, which are discussed in Section 3. The results of the experiment, using multi-agent simulation in order to evaluate our prototype of the disaster evacuation system, are presented in Section 4. The result of attitude survey are described in Section 5, and we describe related work in Section 6.

\section{Disaster Evacuation Assistance System}

\subsection{Issues Relating to Disaster Evacuation}

To facilitate evacuation when a disaster occurs, information on the current situation of each site is useful; hence, some disaster information-sharing systems have been proposed [3][4]. Knowing "which evacuation route is safer" (using evacuation support systems or not), the next issue is "how to evacuate safely and effectively." However, smooth and effective evacuation is not always easy. The provision of additional, appropriate information concerning evacuation guidance may make it feasible to avoid congestion, and successfully shorten evacuation times.

Usually, disaster countermeasure offices are set up in organizations, in order to determine and provide appropriate evacuation routes. However, it is not always easy to plan and provide appropriate information rapidly. In addition, such countermeasure offices have limited resources and they are insufficient for guiding the appropriate timing for evacuation to the evacuees. An effective disaster evacuation assistance system must cope with these issues.

\subsection{Overview of the Disaster Evacuation Assistance System}

\section{A. The Premise of Mobile devices}

The authors propose a disaster evacuation assistance system based on mobile devices. In order to meet the requirements of the system, the following premises apply to the mobile devices:

A-1 Locations of the evacuees: Determine people's individual locations via GPS, or their relative position from Wi-Fi based stations.

A-2 Ad-hoc communication: Neighboring mobile devices can communicate with each other through an ad-hoc communication function.

A-3 Knowledge about the evacuation routes: Mobile devices have knowledge about the evacuation destinations that are appropriate from the current position of each evacuee and their evacuation route. Mobile devices also have the function of path planning

This system requires only mobile devices as the platform; the system does not require any central server.

\section{B. The Requirements for Evacuation Guidance}

The evacuation assistance system proposed in this paper has functions corresponding to the following guidance: 
B-1 Information sharing about the safeness of the evacuation routes: Information on obstacles on each evacuation route is shared through ad-hoc communication between mobile devices. This is the same as discussed in reference [4].

B-2 Planning of the evacuation route: The mobile devices estimate their positions and can make a plan for an evacuation route.

B-3 Negotiation: The appropriate evacuation start time for each evacuee is negotiated by using DCOP with neighbors.

B-4 Manifestation of evacuation guidance: A mobile device presents user evacuation guidance information through a screen, a sound, etc.

In this research, the system is based on the premises described in A-1 to A-3. The negotiation of evacuation timing described in point B-3 is the focus of this research. In order to support evacuation at the time of a disaster, the framework of the distributed constraint optimization problem (DCOP) is effective in solving a problem without using a central server [5]. The DCOP framework is used for adjusting the evacuation timing.

\section{Evacuation Procedure}

The procedure for evacuation using the system we are proposing is given below. When a disaster occurs, the mobile devices shall operate as follows:

C-1 Starting of application: Application is started by the user.

C-2 Acquisition of position information: A mobile device automatically acquires the position it is near. It does not need to be exact, but it needs to decide in which room it is.

C-3 Formation of a group: A group is created for every room. Those who are located in the same room at the evacuation start time belong to the same group.

C-4 Leader finding: The leader of a group is determined. A classic leader finding distribution algorithm is performed by communication between devices. There are no conditions for a leader; any device may become a leader. Supposing a device has an identification number, the device with the lowest identification number may become a leader.

C-5 Planning of an evacuation route: A leader device searches the evacuation route from his position on a map, and draws up an evacuation plan.

C-6 Negotiation: A leader device communicates with a neighboring leader device and exchanges information on an evacuation route. If the same course is used for the same time, it is decided which group has used an evacuation route previously. The framework of DCOP is used for this problem solving.

C-7 Evacuation start: A group with previous experience starts evacuation. The next group negotiates again after a particular period of time.

\section{Formalization of Evacuation}

In this section, the formalization of the problem is described. The type of evacuation covered in this paper is a local adaptation evacuation; the planned evacuation of a wide area is not considered, because this system is based on local area ad-hoc communication and limited computer resources. 


\subsection{Formalization based on DCOP}

In order to use the framework of DCOP, it is necessary to formalize the disaster evacuation problem. Thus, we considered the formalization as follows. An evacuation route is regarded as a resource. This resource assignment will be solved by DCOP.

Evacuees with a mobile device are considered to be agents $A=\left\{a_{1}, \ldots, a_{N}\right\}$. $\mathscr{P}=$ $\cup\left\{p_{1}, \ldots, p_{M}\right\}$ are the places from which people must evacuate. When agent $a_{l}$ is located in time $t$ at place $p_{i}$, it is recorded as place $\left(a_{l}, t\right)=p_{i}$. It entails a large cost for agents $a_{l}$ and $a_{k}$ to be located in the same place at time $t$.

$$
g\left(a_{l}, a_{k}, t\right):= \begin{cases}1, & \text { for } \operatorname{place}\left(a_{l}, t\right) \neq \operatorname{place}\left(a_{k}, t\right) \\ C, & \text { for } \operatorname{place}\left(a_{l}, t\right)=\operatorname{place}\left(a_{k}, t\right)\end{cases}
$$

where $C>1 . G(t)$ is the fundamental constraint of an agent's move. Let $G(t)$ be the summation of these.

$$
G(t)=\sum_{a_{l}, a_{k} \in A} g\left(a_{l}, a_{k}, t\right)
$$

At the time of a disaster, the situation is fluid. People may be unable to pass along a passage. All places $p_{i}$ have a score of $\operatorname{val}\left(p_{i}\right) \in \mathbb{N}$. This score decreases toward the refuge direction. An agent can search the evacuation routes and calculate the scores for the areas on the routes. In this paper, the score allocation for all agents is the same, for simplification purposes. At this time, the utility function (cost function) $f$ for all agents is as follows:

$$
f(A, t):=G(t) \times \sum_{\left\{a_{i} \mid \operatorname{place}\left(a_{i}, t\right) \in \mathscr{P}\right\}} \operatorname{val}\left(\operatorname{place}\left(a_{i}, t\right)\right)
$$

The summation of val(place) expresses the desire to evacuate, and $G(t)$ is a constraint to avoid congestion. It is better that the value of $f$ is small.

In this study, evacuation is considered to be a real-time planning problem. The position after the movement of an agent in time $t+1$ may not be the position that the system computed at time $t$. An agent may be unable to move, depending on the situation, which a system cannot know, or an agent may not follow the guidance of a system. That is, the evacuation problem at time $t$ is to solve the following expression.

$$
\underset{A}{\operatorname{minimize}} f(A, t+1)-f(A, t)
$$

The above $f(A, t+1)-f(A, t)$ is negative. By solving this expression, the agent knows the appropriate evacuation timing. To solve this, we adopt the DCOP framework.

Distributed Constraint Optimization Problems (DCOP) are the fundamental framework in distributed artificial intelligence and have recently attracted considerable attention [2]. Algorithms used to solve DCOP include ADOPT,OptAPO,DPOP,NCBB,DSA, and so on [2]. As for a complete algorithm, an optimum solution is guaranteed, despite the extended computing time. When using DCOP for real-world problems, particularly when solving problems involving robotics and sensor networks, problems must be solved in distributed environments with minimal computation resources [6][7]. Under such circumstances, seeking an optimum solution with a complete algorithm is not always the best method, and there is a need for a fast and efficient approximation algorithm. The framework of DCOP does not need a center server in order to solve problems. 
When treated as a distributed constraint optimization problem, the evacuation problem can be considered as follows.

An agent has a variable to store the place to which the agent should move. The agent also decides the position at time $t+1$ using DCOP at time $t$. This is continuously performed as a real-time planning problem. The situation confirmation for every step is indispensable. At this time, minimization of Formula(3), i.e. minimization of Formula(4), is the objective function. $G(t)$ (Formula (3)) is the constraint of an agent's move and can be described by a binomial constraint.

\subsection{Application}

In this paper, we assume the evacuation situation to be in the campus buildings of a university. When a disaster occurs, it is difficult to grasp how many people there are in a campus building. People in a university are not organized like people in a company. Since it is difficult to control such people's evacuation, guidance from a central office, the guidance system which we propose, is effective.

In the case of evacuation from a campus building, the units of place are classrooms, passages, and stairs. The evacuees use the system to judge whether they should stop at the present classroom or go into a passage. The system adjusts so that a passage does not become congested.

Mobile devices, such as a smart phone, are commonly used these days for noting information about classes (e.g. about examinations, reports, lecture cancellations). This evacuation assistance system is assumed to be implemented as one of the software applications at a university. As regards evacuees who may not have such mobile devices (older people, children), we assume this issue could be resolved by organizing evacuee groups, and evacuees with mobiles could input data about the number of evacuees in the group.

Activation of the system is considered as follows: If more than a fixed number of terminals starts a system simultaneously at a certain place, the system assumes that a disaster has occurred. Then a system wake-up message is sent to a neighboring terminal. The terminal which receives the message starts the evacuation assistance system automatically.

\section{Experiment using Multi-agent Simulation}

Multi-agent simulation [8] is often used for disaster evacuation experiments [9] [10][11], not only for disaster prevention planning, but also for building or city planning. We conducted an experiment using multi-agent simulation in order to investigate the validity of the proposed system.

In any of the following experiments, evacuees move rationally to an evacuation place and they move according to the model of crowd walking [12].

The evacuee's psychological model is not used in this experiment; the evacuee shall follow the guidance if guidance is available. The simulation assumes evacuation from the campus building. Evacuation is started from the situation where people are randomly distributed throughout the classrooms and passages. The evacuation guidance in this experiment negotiates only the evacuation start timing from each classroom by DCOP. If the population density of a passage drops below the threshold value, evacuation will be started from any one classroom on each floor. DCOP shall be used for the selection of the classroom from which evacuation may be started. In this experiment, in order to solve DCOP, the approximation algorithm DSA [6] was used. 


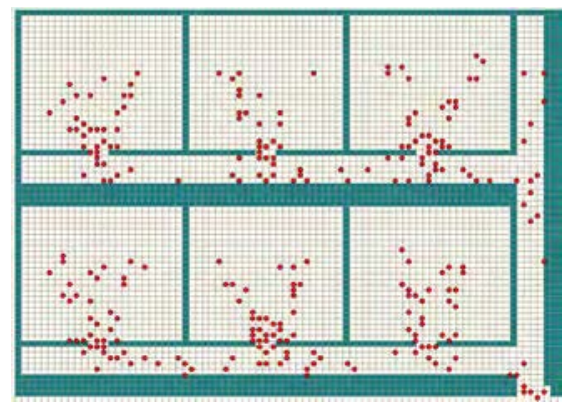

Figure 1: Multi-agent simulation of a disaster evacuation: simple situation

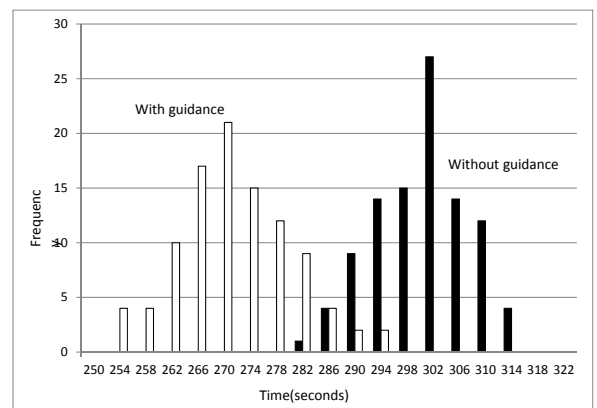

Figure 2: Frequency distribution of the Figure 3: Frequency distribution of the evacuation completion time (with/without evacuation completion time (approximate guidance)

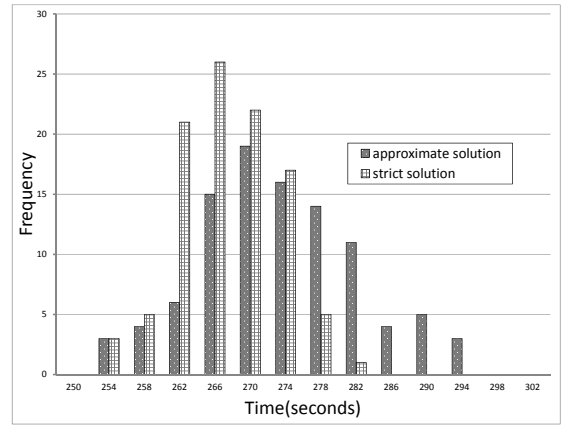

solution/strict solution)

It is important to solve the problem in a short time in an urgent situation such as disaster evacuation. In such a case, an approximation algorithm is more suitable than a complete algorithm. DSA is a randomized algorithm. If the same conditions applied, this decision would be made at random.

In the first experiment, we set up a two-story campus building with six rooms for the experimental conditions. On the map of this experiment, the number of evacuation routes was one. The number of agents was set to 400. The location of the classrooms and the simulation image are shown in Fig. 1.

We compared the evacuation completion times in the cases with and without evacuation guidance. If there is no evacuation guidance, evacuation will be simultaneously started from all the classrooms, but if there is evacuation guidance, evacuation will be started from one classroom.

The simulation was performed 300 times under these conditions. The resulting frequency distribution of the evacuation completion time of all evacuees is shown in 2 . The evacuation completion time was plotted on the $\mathrm{X}$-axis, and frequency was plotted on the Y-axis. In this experimental result, when there was evacuation guidance by the system, evacuation completion time decreased by about $10 \%$. This effect should change according to the conditions, such as the location of the classrooms, the width of the passage, and so on.

The approximation algorithm DSA was used for this experiment. For this reason, al- 


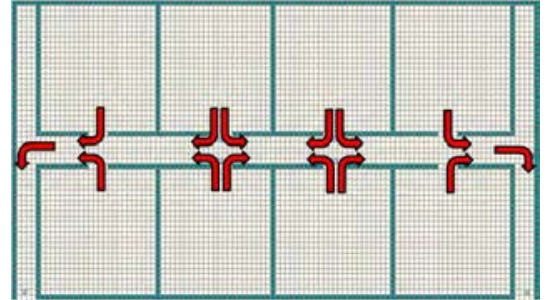

Figure 4: Multi-agent simulation of a disaster evacuation: two evacuation routes

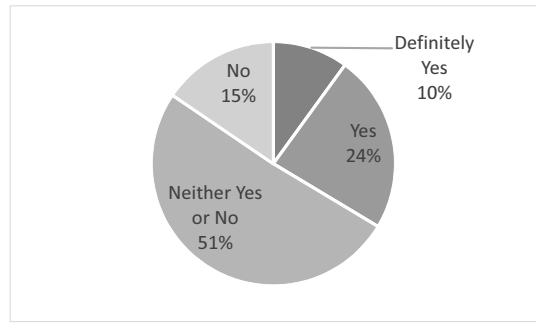

Figure 6: The attitude survey result(1)

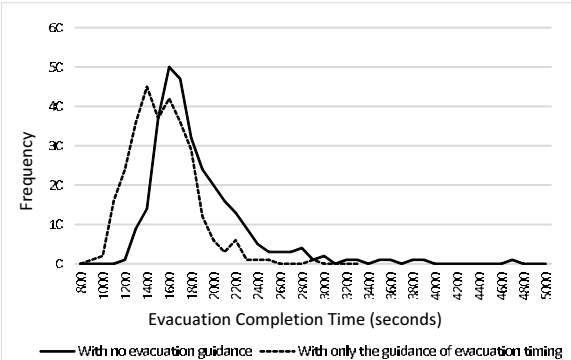

Figure 5: Frequency distribution of the evacuation completion time (long-tailed)

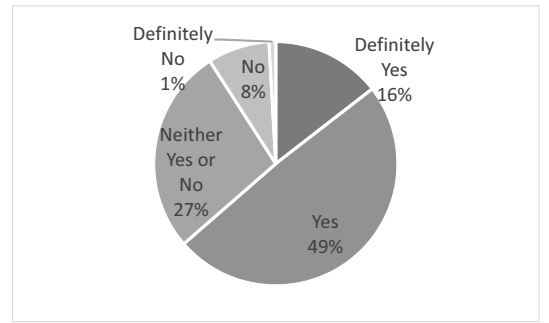

Figure 7: The attitude survey result(2)

though evacuation should ideally have been performed from any one classroom on each floor, evacuation might have been simultaneously performed from two classrooms. This is a case where a strict solution cannot be found by DSA.

The case where evacuation is performed only from one classroom is called a strict solution. The frequency distribution of only a strict solution is shown in Fig. 3. In this figure, the case of the approximate solution was also plotted for comparison. Although a significant difference was observed between these two as a result of a $t$-test, the difference was very slight. In this experiment, there were only three classrooms on one floor, so the difference between the strict solution and the approximate solution might be small.

As the next step of the experiments, we experimented using a map that can choose two evacuation routes. The map is shown in Fig. 4. The number of agents was set to 700. From four central rooms, progress can be made in both of the two directions after coming out into a passage. Both sides were stairs. In the simulation, we set up a rule that the walking speed of an evacuee slowed when passing through stairs. This is a part of the large complicated map.

In this experiment, the evacuees who came out of these rooms into the passage chose either one of the two staircases with a ratio of 60:40. (Nearby stairs accounted for 60\%.) The simulation was performed 300 times under each condition.

In this experiment, when there was no evacuation guidance, great confusion was observed in the vicinity of the center of the passage. When confusion occurs, the evacuation completion time might become very long. The frequency distribution of the evacuation completion time of the experimental result is shown in Fig. 5. The distribution is longtailed when there was no evacuation guidance. As shown in Fig. 5, this tail is short if there was guidance on evacuation timing. Accidents, such as a fall, may occur due to such confusion. The guidance system is effective in order to avoid such confusion. 


\section{Survey on Consciousness of Evacuation Guidance}

Making people follow guidance may be one of the most important issues affecting an evacuation system, because it will affect the effectiveness of the evacuation. To understand the extent to which people will obey evacuation guidance exactly, conducting an experiment with a huge number of experimental evacuees would be desirable. However, this would not be realistic, especially in the requirement analysis phase. Therefore, the authors conducted an attitude survey. Data collection took place as internet survey in November, 2014. Totally, 110 samples were collected.

Fig. 6 shows the answer for the question "Do you think you could wait according to the evacuation guidance, even if you did not have absolute proof that other people would obey the system?". It seems to be effective if evacuees can recognize that other people will also follow the system eachother. Fig. 7 shows the answer for the question. "Do you think you could wait according to the evacuation guidance, if you recognized that your waiting would contribute to the overall efficiency of the evacuation?", and the answer shows that, by understanding their contribution to the overall evacuation, many people could wait for a while. From the survey results, by understanding their contribution to the overall evacuation, more than $60 \%$ of people could wait for a while. Clarifying the effect of waiting would motivate people to follow the system, and wait.

\section{Related Work}

In [3] and [4], disaster information sharing systems are proposed. This research has not discussed evacuation timing. Our research has focused on evacuation timing. We use distributed processing (DCOP) in order to calculate a solution, and no central server was needed. However, it will be necessary to mutually complement this research in order to realize the evacuation assistance system.

[14] discussed the application of DCOP for coordination in a disaster management situation. Authorities must assign tasks and resources in disaster scenarios; unfortunately, accomplishing this in real time is currently difficult. They argue that the framework of DCOP is uniquely suited to meet the requirements imposed on coordination mechanisms in these settings. [5] extended DCOP to Stochastic DCOP(SDCOP) in order to apply it to disaster management, as proposed by [14]. In SDCOP, the constraint rewards are deterministic values but are sampled from known probability distribution functions called reward functions. They proposed an algorithm that solves SDCOP. This research specializes in the resource (or shelter) assignment problem at the time of a disaster, and has not made reference to evacuation guidance. Since the purpose of this research is algorithm development for SDCOP, it has not mentioned a detailed application. We used the simple DSA algorithm in order to solve DCOP. We may consider the use of the algorithm for SDCOP in the future, in order to solve the problem of complicated conditions.

Evacuation problems can be modeled in dynamic network flows [15]. The standard approach to solving dynamic flow problems is to transform the graph into a time-expanded network. However, the expanded graph is large. The major computational bottlenecks are the time and memory required to construct the expanded network. Some heuristic algorithms have been proposed for this problem [15]. [16] considered capacity constrained routing heuristics. [17] considered the problem of planning evacuation routes in deteriorating networks, where nodes become unavailable over time. In this research of heuristic 
algorithms, it is assumed that problem solving is performed in a non-distributed environment. Our research is incompatible with this, since such calculation systems need a central server.

There is also some related work concerning disaster evacuation simulation systems using multi-agent simulation [9][10][11].

\section{Conclusions}

We aimed to develop a system that provides optimal evacuation guidance autonomously at the time of a disaster. This system enables assistance to be given in the form of evacuation guidance to relieve congestion, by calculating evacuation routes and timing via an ad-hoc network of evacuees' mobile devices, without a central server.

In this paper, the problem of disaster evacuation was formalized and we examined how to solve it using the framework of the distributed constraint optimization problem. In the experiments using multi-agent simulations, when there was evacuation guidance using DCOP, the evacuation completion time decreased by about 10\%-30\%. Even when it was solved with an approximation algorithm, the effect on the evacuation completion time was small.

One of the characteristics of the system proposed in this paper is the control of traffic congestion during disaster evacuation. This system is fundamentally applicable to various scenarios. At present, the prototype of the system we are proposing in this paper is based on a simple case in university campuses. However, adding other functions based on various use-cases will provide a broader coverage for the effectiveness of this system.

\section{Acknowledgements}

This work was supported in part by a JSPS Grant-in-Aid for Scientific Research (25350481).

\section{References}

[1] CabinetOffice, Disaster Management In Japan. Government of Japan, 2011. [Online]. Available: http://www.bousai.go.jp/1info/pdf/saigaipanf_e.pdf

[2] A. R. Leite, F. Enembreck, and J.-P. A. Barthes, "Distributed constraint optimization problems: Review and perspectives," Expert Systems with Applications, vol. 41, no. 11 , pp. $5139-5157,2014$.

[3] K. Iizuka, Y. Iizuka, and K. Yoshida, "A real-time disaster situation mapping system for university campuses," in Online Communities and Social Computing, ser. Lecture Notes in Computer Science, A. Ozok and P. Zaphiris, Eds. Springer Berlin / Heidelberg, 2011, vol. 6778, pp. 40-49.

[4] A. Fujihara and H. Miwa, "Effect of traffic volume in real-time disaster evacuation guidance using opportunistic communications," in Intelligent Networking and Collaborative Systems (INCoS), 2012 4th International Conference on, Sept 2012, pp. 457-462. 
[5] D. T. Nguyen, W. Yeoh, and H. C. Lau, "Stochastic dominance in stochastic dcops for risk-sensitive applications," in Proceedings of the 11th International Conference on Autonomous Agents and Multiagent Systems-Volume 1. International Foundation for Autonomous Agents and Multiagent Systems, 2012, pp. 257-264.

[6] W. Zhang, G. Wang, Z. Xing, and L. Wittenburg, "Distributed stochastic search and distributed breakout: properties, comparison and applications to constraint optimization problems in sensor networks," Artif. Intell., no. 161, pp. 55-87, 2005.

[7] S. Fitzpatrick and L. Meertens, "An experimental assessment of a stochastic, anytime, decentralized, soft colourer for sparse graphs," in 1st Symposium on Stochastic Algorithms: Foundations and Applications, 2001, pp. 49-64.

[8] C. M. Macal and M. J. North, "Tutorial on agent-based modeling and simulation," in Proceedings of the 37th Conference on Winter Simulation, ser. WSC '05. Winter Simulation Conference, 2005, pp. 2-15.

[9] C. Burstedde, K. Klauck, A. Schadschneider, and J. Zittartz, "Simulation of pedestrian dynamics using a two-dimensional cellular automaton," Physica A: Statistical Mechanics and its Applications, vol. 295, no. 3, pp. 507-525, 2001.

[10] D. Helbing, I. Farkas, and T. Vicsek, "Simulating dynamical features of escape panic," Nature, vol. 407, no. 6803, pp. 487-490, 2000.

[11] J. Shi, A. Ren, and C. Chen, "Agent-based evacuation model of large public buildings under fire conditions," Automation in Construction, vol. 18, no. 3, pp. 338-347, 2009.

[12] T. Kaneda and D. Okayama, "A pedestrian agent model using relative coordinate systems," in Agent-Based Approaches in Economic and Social Complex Systems IV. Springer, 2007, pp. 63-70.

[13] K. Iizuka and Y. Iizuka, "Consideration of behavioral psychological issues for the mobile evacuation guidance systems," in The Inaugural Asian Conference on the Social Sciences and Sustainability, 2014, pp. 109-114.

[14] R. N. Lass, J. B. Kopena, E. A. Sultanik, D. N. Nguyen, C. P. Dugan, P. J. Modi, and W. C. Regli, "Coordination of first responders under communication and resource constraints," in Proceedings of the 7th international joint conference on Autonomous agents and multiagent systems-Volume 3. International Foundation for Autonomous Agents and Multiagent Systems, 2008, pp. 1409-1412.

[15] H. W. Hamacher and S. A. Tjandra, "Mathematical modelling of evacuation problems-a state of the art," Pedestrian and evacuation dynamics, vol. 2002, no. 227266, pp. 1-2, 2002.

[16] Q. Lu, B. George, and S. Shekhar, "Capacity constrained routing algorithms for evacuation planning: A summary of results," in Advances in spatial and temporal databases. Springer, 2005, pp. 291-307.

[17] T. Hadzic, K. N. Brown, and C. J. Sreenan, "Real-time pedestrian evacuation planning during emergency," in Tools with Artificial Intelligence (ICTAI), 2011 23rd IEEE International Conference on. IEEE, 2011, pp. 597-604. 